\title{
Comparative effects of testosterone propionate, oestradiol benzoate, ICI 182,780, tamoxifen and raloxifene on hypothalamic differentiation in the female rat
}

\author{
L Pinilla, M L Barreiro, L C Gonzalez, M Tena-Sempere \\ and E Aguilar
}

Department of Cell Biology, Physiology and Immunology, University of Córdoba School of Medicine, Córdoba, Spain

(Requests for offprints should be addressed to E Aguilar; Email: fi1agbee@uco.es)

\begin{abstract}
Hypothalamic differentiation in the female rat during the neonatal period is critically dependent on the steroid milieu, as permanent changes in reproductive function are observed after administration of oestradiol and testosterone during such a critical stage. Selective oestrogen modulators (SERMs) constitute a family of drugs that, depending on the tissue, are able to exert oestrogenic or antioestrogenic actions. The present experiments were conducted to analyse whether the SERMs, tamoxifen and raloxifene, can cause oestrogenic actions during the hypothalamic differentiation period.

Postnatal female rats were injected between days 1 and 5 with $100 \mu \mathrm{g} /$ day tamoxifen, raloxifene or ICI 182,780 (a pure antioestrogen). Other groups of animals were injected on day 1 of age with $100 \mu \mathrm{g}$ oestradiol benzoate $(\mathrm{OeB})$ or $1.25 \mathrm{mg}$ testosterone propionate (TP) alone or in combination with raloxifene (500 $\mu \mathrm{g} /$ day between days 1 and 5$)$. In all experimental groups, the age, body weight and concentrations of serum gonadotrophins at vaginal opening were recorded, whereas vaginal cyclicity and the negative and positive feedback between oestradiol and LH were monitored in adulthood.

The results obtained confirmed the ability of high doses of $\mathrm{OeB}$ or TP to alter the normal differentiation of the
\end{abstract}

brain permanently. They also reinforced the hypothesis that oestrogens are also necessary for normal brain differentiation in female rats because administration of a pure antioestrogen, such as ICI 182,780 permanently altered the function of the reproductive axis. In addition, our data provided evidence for different actions of the two SERMs under analysis (raloxifene and tamoxifen) upon peripheral targets, as raloxifene advanced vaginal opening whereas tamoxifen did not. In contrast, their actions on brain differentiation appeared similar and analogous to those obtained after neonatal administration of oestradiol, as evidenced by vaginal acyclicity, ovarian atrophy, sterility and abolition of negative and positive feedback between oestradiol and LH, thus suggesting an oestrogenic action of these SERMs on hypothalamic differentiation. Moreover, the oestrogenic activity of raloxifene was supported by its inability to block the effects of $\mathrm{OeB}$ and $\mathrm{TP}$ administered neonatally.

In conclusion, the present results indicated that the SERMs, tamoxifen and raloxifene, exert an oestrogen-like effect upon hypothalamic differentiation of the neonatal female rat.

Journal of Endocrinology (2002) 172, 441-448

\section{Introduction}

The oestrogen receptor modulators (SERMs) have been developed for improving the management of diseases associated with menopausal women. Tamoxifen, originally developed as an oestrogen antagonist and widely used for the treatment of breast cancer (Jordan \& Morrow 1999), has been found to act as a partial oestrogen agonist in the uterus and to display oestrogen agonist effects in bone and in the cardiovascular system (Jordan \& Murphy 1990, Jordan 1993, Jordan \& Morrow 1999). More recently, a new class of SERMs has been developed. Among them, raloxifene has been found to block the effects of oestrogen in the breast whereas it mimics oestrogen action on bone and blood lipid concentration (Wakeling et al. 1984, Glasebrook et al. 1993, Yang et al. 1996, Delmas et al. 1997).

In the rodent central nervous system (CNS), steroid input on hypothalamic neurones immediately after birth is critical for the organization of the neural mechanisms involved in the control of the neuroendocrine function, especially those serving reproductive activity (Barraclough 1961, Gorski 1963, Dhar \& Setty 1976, Aguilar et al. 1979, Pinilla et al. 1993). The effects of SERMs in the 
organization of the neural circuitry controlling reproductive function have not yet been evaluated extensively. Early postnatal treatment of female rats with tamoxifen resulted in permanent anovulatory sterility (Hancke \& Döhler 1980). The fact that this effect was partially prevented by simultaneous treatment with oestradiol supported the idea that tamoxifen acts as an antioestrogen in the CNS of neonatal female rats, and that a certain degree of oestrogenic input is necessary for normal female differentiation of the brain (Brown-Grant 1974, Hancke \& Döhler 1980). Recently, we have suggested that neonatal administration of raloxifene induces permanent alterations that were similar to those obtained after exposure to oestradiol (Pinilla et al. 2001a) in the reproductive function of the female rat.

The present experiments were carried out to compare the effects of neonatal administration of oestradiol, testosterone, ICI 182,780 (an antioestrogen devoid of oestrogenic activity) (Wakeling et al. 1991, Wakeling \& Bowler 1992, Wade et al. 1993), tamoxifen and raloxifene (SERMs with compound oestrogenic/antioestrogenic activities) in the organization of the neural network involved in the control of the hypothalamic-pituitaryovarian axis. In addition, we tested the ability of raloxifene to counteract the deleterious effects of neonatal exposure to oestradiol and testosterone on female reproductive function. We have previously proposed (Pinilla et al. $2001 b)$ that raloxifene may act in adulthood as an antioestrogen depending on the prevailing oestrogenic background. If this hypothesis is true, the effects of oestradiol or testosterone, which increase (directly or after testosterone aromatization) oestradiol input to the hypothalamus, should be reduced in neonatal rats.

\section{Materials and Methods}

\section{Animals and treatments}

Female Wistar rats born in our laboratory were kept under controlled conditions of light (12 h light: $12 \mathrm{~h}$ darkness, lights on at $0700 \mathrm{~h})$ and temperature $\left(22^{\circ} \mathrm{C}\right)$, and had free access to pelleted food (Pacsa Sanders, Seville, Spain) and tap water. The day on which the litters were born was considered to be day 1 of age. At this stage, the litter size was adjusted to eight animals. These animals were weaned on day 21 and housed in groups of four to five animals per cage. Raloxifene chlorhydrate (2-(4-hydroxyphenyl)-6hydroxybenzo-b-thien-3-yl-[4]-2-(1-piperidinyl)ethoxyphenyl-methanone hydrochloride; Evista) was obtained from Lilly, Basingstoke, Hants, UK. The pure antioestrogen ICI 182,780 (7-[9-[4,4,5,5,5-penthafluoropentyl)sulphinil]nonyl]-estra-1,3,5(10)-triene-3,17-diol) was purchased from Tocris (Madrid, Spain). Tamoxifen, $17 \beta$-oestradiol 3-benzoate $(\mathrm{OeB})$ and testosterone propionate (TP) were purchased from Sigma (Barcelona, Spain). Raloxifene chlorhydrate was dissolved in saline, OeB and TP were dissolved in olive oil and tamoxifen and ICI 182,780 were dissolved initially in a few drops of dimethylsulphoxide and ethanol. Thereafter, they were dissolved in saline up to the working concentration; the injection volume was $0 \cdot 1 \mathrm{ml}$.

\section{Experimental designs}

Female rats were injected i.p. between days 1 and 5 of age with tamoxifen $(100 \mu \mathrm{g} / \mathrm{rat}$ per day), raloxifene $(100 \mu \mathrm{g} /$ rat per day), ICI $182,780(100 \mu \mathrm{g} /$ rat per day $)$ or vehicle. Other groups of female rats were injected s.c. on day 1 of age with OeB $(100 \mu \mathrm{g} / \mathrm{rat})$, TP $(1.25 \mathrm{mg} / \mathrm{rat})$ or olive oil and between days 1 and 5 with vehicle or raloxifene $(500 \mu \mathrm{g} / \mathrm{rat}$ per day). The age and body weight at vaginal opening were recorded. On this day, blood samples were collected after light ether anaesthesia. After vaginal opening, vaginal smears were taken each day. Abnormal cycles were considered to be those showing more than 14 consecutive days of oestrous or dioestrous phases. Animals were ovariectomized on day 90, and 14 days later were injected i.p. with $75 \mu \mathrm{g} \mathrm{OeB}$. Blood samples were obtained by jugular venipuncture after light ether anaesthesia before ovariectomy and on days 1, 7, 14 (at $1000 \mathrm{~h}$ ), 15 and 16 (at 1000 and $1800 \mathrm{~h}$ ) after ovariectomy. The experiment was approved by the Córdoba University Ethical Committee for animal experimentation and was conducted in accordance with the European Union regulation governing the care and use of experimental animals.

\section{Hormone assays}

Serum concentrations of luteinizing hormone (LH) and follicle-stimulating hormone (FSH) were measured by specific radioimmunoassays using kits supplied by the NIDDK (Bethesda, MD, USA), and hormone concentrations were expressed using LH-RP-3 and FSH-RP-2 as standards. All samples from each experiment were measured in the same assay. Intra-assay coefficients of variation were $<8 \%$ and the sensitivities of the assays were 20 and $7 \cdot 5 \mathrm{pg} / 50 \mu \mathrm{l}$ for $\mathrm{LH}$ and FSH respectively.

\section{Statistical analysis}

Data are expressed as means \pm S.E.M. Chi-squared evaluation was used selectively for comparing data on the percentages of abnormal cycles. All other data were analysed by one- or two-way ANOVA followed by Tukey's test.

\section{Results}

Effects of neonatal OeB, TP, tamoxifen, raloxifene and ICI 182,780 on pubertal presentation (Table 1)

Vaginal opening was advanced in female rats treated neonatally with $\mathrm{OeB}$, raloxifene or both, while tamoxifen 
Table 1 Age, body weight and serum concentrations of $\mathrm{LH}$ and $\mathrm{FSH}$ on the day of vaginal opening in rats treated with tamoxifen $(100 \mu \mathrm{g} / \mathrm{rat}$ per day), raloxifene $(100$ and $500 \mu \mathrm{g} / \mathrm{rat}$ per day), ICI 182,780 (100 $\mu \mathrm{g} /$ rat per day) or vehicle on days $1-5$ of age and rats treated with oestradiol benzoate (OeB) $(100 \mu \mathrm{g} / \mathrm{rat}$ per day) or oil on day 1 of age and raloxifene $(500 \mu \mathrm{g} / \mathrm{rat}$ per day) or vehicle on days $1-5$ of age. Data are expressed as means \pm S.E.M. (9-13 animals per group)

\begin{tabular}{|c|c|c|c|c|}
\hline & $\begin{array}{l}\text { Age } \\
\text { (days) }\end{array}$ & $\begin{array}{l}\text { Body weight } \\
\text { (g) }\end{array}$ & $\begin{array}{l}\text { LH } \\
(\mathrm{ng} / \mathrm{ml})\end{array}$ & $\begin{array}{l}\text { FSH } \\
(\mathrm{ng} / \mathrm{ml})\end{array}$ \\
\hline \multicolumn{5}{|l|}{ Treatment } \\
\hline Vehicle & $38 \cdot 1 \pm 1 \cdot 0$ & $131 \cdot 6 \pm 4 \cdot 7$ & $0 \cdot 65 \pm 0.05$ & $4 \cdot 6 \pm 0 \cdot 8$ \\
\hline Tamoxifen & $40 \cdot 8 \pm 0 \cdot 4^{\mathrm{a}}$ & $131 \cdot 8 \pm 3 \cdot 1^{\mathrm{a}}$ & $0 \cdot 57 \pm 0.07^{a}$ & $5 \cdot 6 \pm 0 \cdot 5$ \\
\hline Raloxifene (100) & $25 \cdot 3 \pm 0 \cdot 4^{* *}$ & $60 \cdot 5 \pm 2 \cdot 3^{* *}$ & $0 \cdot 21 \pm 0.02^{* *}$ & $3 \cdot 8 \pm 0 \cdot 4$ \\
\hline Raloxifene (500) & $17 \cdot 0 \pm 0 \cdot 1^{* *}$ & $27 \cdot 3 \pm 0 \cdot 6^{* *}$ & $0 \cdot 25 \pm 0 \cdot 1^{* *}$ & $14 \cdot 7 \pm 1 \cdot 4^{* *}$ \\
\hline ICI 182,780 & $39 \cdot 8 \pm 0 \cdot 6$ & $136 \cdot 5 \pm 5 \cdot 1$ & $0 \cdot 92 \pm 0 \cdot 1$ & $5 \cdot 5 \pm 0 \cdot 7$ \\
\hline Oil+vehicle & $34 \cdot 4 \pm 0 \cdot 7$ & $120 \cdot 7 \pm 4 \cdot 5$ & $0 \cdot 87 \pm 0 \cdot 2$ & $6 \cdot 8 \pm 1 \cdot 7$ \\
\hline OeB + vehicle & $17 \cdot 1 \pm 0 \cdot 1^{* *}$ & $31 \cdot 2 \pm 0 \cdot 9$ ** & $0 \cdot 17 \pm 0.04^{* *}$ & $3 \cdot 0 \pm 0 \cdot 4^{* *}$ \\
\hline OeB + raloxifene & $17 \cdot 0 \pm 0 \cdot 2^{* *}$ & $27 \cdot 5 \pm 0 \cdot 6^{* *}$ & $0 \cdot 17 \pm 0.06^{* *}$ & $8 \cdot 4 \pm 0 \cdot 8^{c}$ \\
\hline
\end{tabular}

${ }^{* *} P \leq 0.01$ vs vehicle; ${ }^{a} P \leq 0.01$ tamoxifen vs raloxifene; ${ }^{c} P \leq 0.01$ OeB + raloxifene vs OeB +vehicle (ANOVA followed by Tukey's test).

Table 2 Percentage or rats showing abnormal cyclicity and ovarian weight in 90-day-old female rats treated with tamoxifen $(100 \mu \mathrm{g} / \mathrm{rat}$ per day), raloxifene (100 and $500 \mu \mathrm{g} / \mathrm{rat}$ per day), $\mathrm{ICl} 182,780(100 \mu \mathrm{g} /$ rat per day) or vehicle on days $1-5$ of age and in rats treated with OeB $(100 \mu \mathrm{g} / \mathrm{rat})$, testosterone propionate (TP) $(1.25 \mathrm{mg} / \mathrm{rat})$ or oil on day 1 of age and raloxifene $(500 \mu \mathrm{g} / \mathrm{rat}$ per day) or vehicle on days $1-5$ of age. Data are expressed as means \pm S.E.M

\begin{tabular}{|c|c|c|c|c|}
\hline & $\begin{array}{l}\text { No. of rats } \\
\text { tested }\end{array}$ & $\begin{array}{l}\% \text { of rats } \\
\text { with abnormal } \\
\text { cycles }\end{array}$ & $\begin{array}{l}\text { Ovarian weight } \\
(\mathrm{mg})\end{array}$ & $\begin{array}{l}\text { Ovarian weight } \\
\text { (mg \% g body weight) }\end{array}$ \\
\hline \multicolumn{5}{|l|}{ Treatment } \\
\hline Vehicle & 11 & 0 & $82 \cdot 4 \pm 5 \cdot 0$ & $27 \cdot 2 \pm 1 \cdot 6$ \\
\hline Tamoxifen & 10 & 100 & $33 \cdot 7 \pm 2 \cdot 0^{* *, a}$ & $11 \cdot 5 \pm 0 \cdot 9^{* *, a}$ \\
\hline Raloxifene (100) & 10 & 100 & $57 \cdot 2 \pm 3 \cdot 2^{* *}$ & $18 \cdot 2 \pm 1 \cdot 1^{\star *}$ \\
\hline Raloxifene (500) & 10 & 100 & $48 \cdot 1 \pm 2 \cdot 8^{* *}$ & $16 \cdot 1 \pm 1 \cdot 4^{* *}$ \\
\hline ICI 182,780 & 13 & 46 & $66 \cdot 6 \pm 3 \cdot 6^{*}$ & $23 \cdot 1 \pm 1 \cdot 4^{*}$ \\
\hline Oil+vehicle & 9 & 0 & $108 \cdot 0 \pm 4 \cdot 0$ & $38 \cdot 2 \pm 0 \cdot 9$ \\
\hline OeB + vehicle & 11 & 100 & $67 \cdot 8 \pm 2 \cdot 4^{* *}$ & $22 \cdot 2 \pm 1 \cdot 0^{\star *}$ \\
\hline TP+vehicle & 11 & ND & $75 \cdot 3 \pm 1 \cdot 4^{* *}$ & $27 \cdot 6 \pm 0 \cdot 8^{* \star}$ \\
\hline OeB +raloxifene & 9 & 100 & $66 \cdot 4 \pm 3 \cdot 3^{* *}$ & $26 \cdot 1 \pm 1 \cdot 1^{* *, c}$ \\
\hline $\mathrm{TP}+$ raloxifene & 11 & ND & $34 \cdot 4 \pm 3 \cdot 4^{* \star, d}$ & $13 \cdot 9 \pm 1 \cdot 7^{* *, d}$ \\
\hline
\end{tabular}

${ }^{*} P \leq 0.05$ vs vehicle; ${ }^{* *} P \leq 0.01$ vs vehicle; ${ }^{\text {a }} P \leq 0.01$ tamoxifen vs raloxifene; ${ }^{\mathrm{c}} P \leq 0.01$ OeB + raloxifene vs OeB+vehicle; ${ }^{d} P \leq 0 \cdot 01 \mathrm{TP}+$ raloxifene vs TP+vehicle (ANOVA followed by Tukey's test).

$\mathrm{ND}$, not detectable.

did not change the age of vaginal opening. In contrast, female rats injected with TP with or without raloxifene did not have vaginal opening (data not shown). Precocious vaginal opening in females treated with $\mathrm{OeB}$ or raloxifene $(100$ or $500 \mu \mathrm{g} /$ day) occurred at a lower body weight and serum LH levels than in the control group. In animals treated with $\mathrm{OeB}$ and raloxifene, vaginal opening took place at a similar age to that in animals treated with OeB alone. Serum FSH levels at vaginal opening were significantly reduced in oestrogenized animals and increased in females injected with $500 \mu \mathrm{g} /$ day raloxifene, whereas animals treated with $\mathrm{OeB}$ and raloxifene showed normal FSH levels.
Effects of neonatal OeB, TP, tamoxifen, raloxifene and ICI 182,780 on vaginal cycles and ovarian weights (Table 2)

All animals neonatally treated with tamoxifen, raloxifene (100 or $500 \mu \mathrm{g} /$ day) and OeB showed altered vaginal cyclicity, which consisted of permanent increases in the rate of vaginal oestrous or dioestrous presentation. In contrast, only $46 \cdot 1 \%$ of the rats treated with ICI 182,780 showed this alteration. In animals treated with TP, analysis of vaginal cyclicity was not possible due to the absence of vaginal canalization. In adults, all experimental groups presented a significant decrease in absolute and relative ovarian weights. 


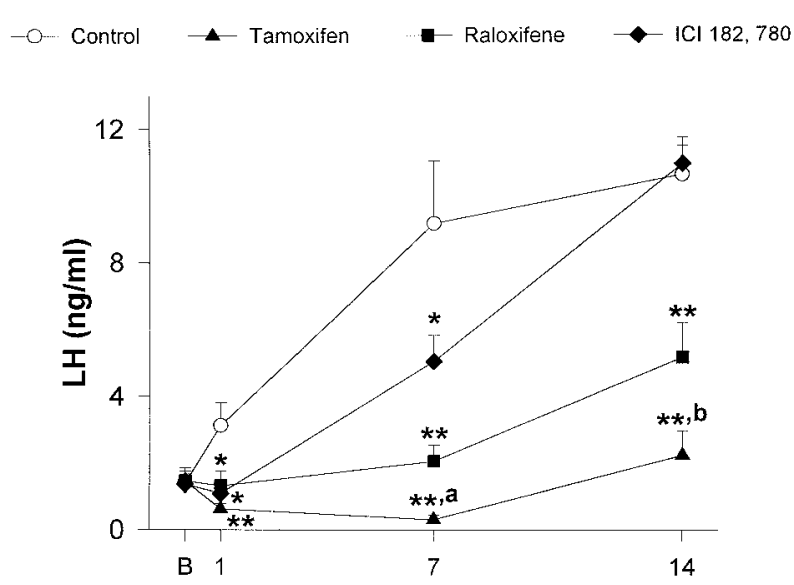

$$
\begin{array}{lll}
-0-\text { Oil }+ \text { Vehicle } & \checkmark \text { OeB }+ \text { Vehicle } & \square-\text { TP }+ \text { Vehicle } \\
-\ldots \text { Oil }+ \text { Rx } & \sim \text { OeB }+ \text { Rx } & \cdots \text { TP }+ \text { Rx }
\end{array}
$$
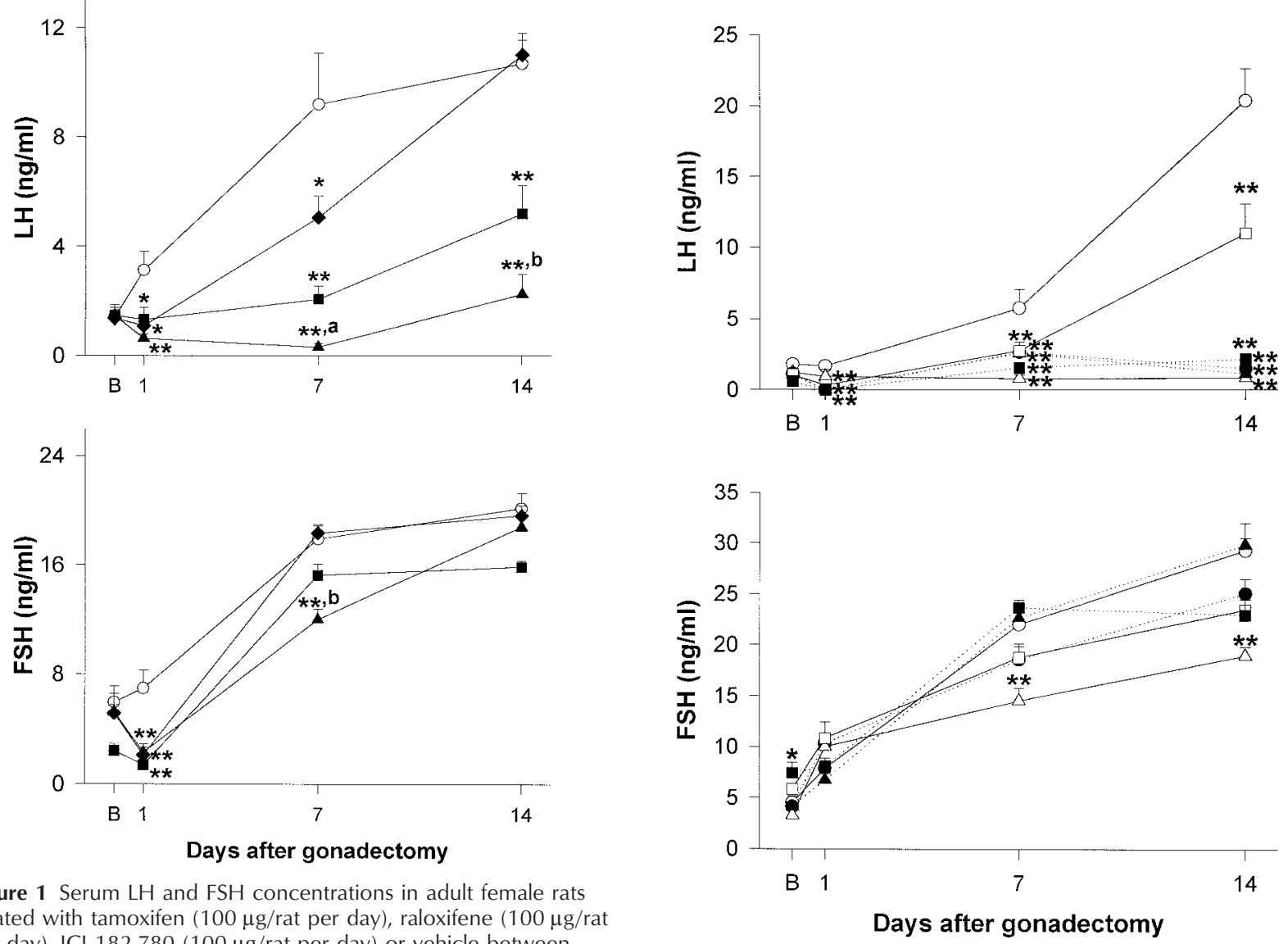

Figure 1 Serum LH and FSH concentrations in adult female rats treated with tamoxifen $(100 \mu \mathrm{g} / \mathrm{rat}$ per day), raloxifene $(100 \mu \mathrm{g} / \mathrm{rat}$ per day), ICI 182,780 (100 $\mu \mathrm{g} /$ rat per day) or vehicle between days 1 and 5 of age. Blood samples were obtained before and 1, 7 and 14 days after ovariectomy. Data are expressed as means \pm S.E.M. ( $10-13$ animals per group). ${ }^{*} P \leq 0.05$ and ${ }^{* *} P \leq 0.01$ vs vehicle; ${ }^{a} P \leq 0.01$ and ${ }^{b} P \leq 0.05$ tamoxifen vs raloxifene (ANOVA followed by Tukey's test).

Effects of neonatal tamoxifen, raloxifene and ICI 182,780 on adult basal and post-ovariectomy serum FSH and LH concentrations (Fig. 1)

Neonatal treatment with tamoxifen, raloxifene or ICI 182,780 did not modify the serum levels of LH and FSH in adults. Treatment with tamoxifen and raloxifene significantly $(P \leq 0 \cdot 01)$ decreased the serum LH concentrations 1,7 and 14 days after ovariectomy, whereas treatment with ICI 182,780 only decreased $(P \leq 0 \cdot 05)$ the LH response 1 and 7 days after ovariectomy; the levels were similar to those of control animals on day 14 post-ovariectomy. FSH levels were lower in all experimental groups than in controls on day 1 after ovariectomy. Afterwards, a significant decrease on day 7 postovariectomy was observed only in animals injected with tamoxifen. Results obtained from animals injected with

$500 \mu \mathrm{g} /$ day raloxifene were similar to those obtained with $100 \mu \mathrm{g}$ (data not shown).

Effects of neonatal raloxifene on adult basal and post-ovariectomy serum FSH and LH concentrations in female rats neonatally injected with $\mathrm{OeB}$ or TP (Fig. 2)

Androgenized animals showed a decrease in the LH response to ovariectomy compared with controls, whether or not they had been treated with raloxifene. Oestrogenized animals showed no increase in plasma LH after ovariectomy and their FSH response was lower than in the control group. In these animals, the combined 

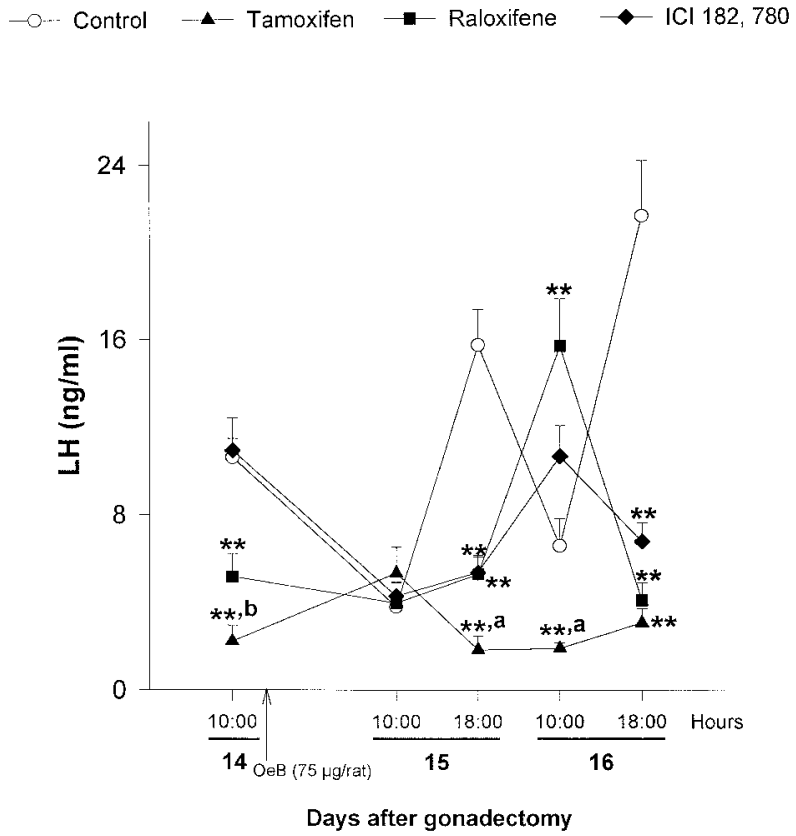

Figure 3 Serum LH concentrations in adult female rats treated with tamoxifen $(100 \mu \mathrm{g} / \mathrm{rat}$ per day $)$, raloxifene $(100 \mu \mathrm{g} /$ rat per day), ICl 182,780 (100 $\mu \mathrm{g} /$ rat per day) or vehicle between days 1 and 5 of age. Blood samples were obtained on different days after ovariectomy (day 14, $1000 \mathrm{~h}$; days 15 and 16, 1000 and $1800 \mathrm{~h}$ ). Animals were injected on day 14 post-ovariectomy with $75 \mu \mathrm{g}$ OeB $(1000$ h). Data are expressed as means \pm S.E.M. $(10-13$ animals per group). ${ }^{* *} P \leq 0.01$ vs control group; ${ }^{a} P \leq 0.01$ and ${ }^{\mathrm{b}} \mathrm{P} \leq 0.05$ tamoxifen vs raloxifene (ANOVA followed by Tukey's test).

administration of raloxifene and oestrogen neonatally normalized only the FSH response to ovariectomy.

Effects of neonatal tamoxifen, raloxifene and ICI 182,780 on adult $\mathrm{LH}$ responses after administration of $75 \mu \mathrm{g} \mathrm{OeB}$ (Fig. 3)

In control female rats, serum concentrations of $\mathrm{LH}$ were higher on day 14 post-ovariectomy than in rats neonatally injected with tamoxifen, raloxifene or ICI 182,780. In control females, administration of $75 \mu \mathrm{g}$ OeB elicited negative and positive feedback actions on LH release. Negative feedback was evidenced by the decrease in serum LH levels at $1000 \mathrm{~h}$, while positive feedback resulted in $\mathrm{LH}$ increases at $1800 \mathrm{~h}$. In tamoxifen-treated rats, administration of $75 \mu \mathrm{g} \mathrm{OeB}$ did not change $\mathrm{LH}$ concentrations at any time studied. In raloxifene-treated rats, there was no evidence of negative feedback and a significant increase was only observed $48 \mathrm{~h}$ after $\mathrm{OeB}$ administration (at $1000 \mathrm{~h}$ of day 16). In ICI 182,780treated rats, negative feedback was shown but positive feedback was absent. Results obtained in animals injected with $500 \mu \mathrm{g} /$ day raloxifene were similar to those obtained with $100 \mu \mathrm{g}$ (data not shown).
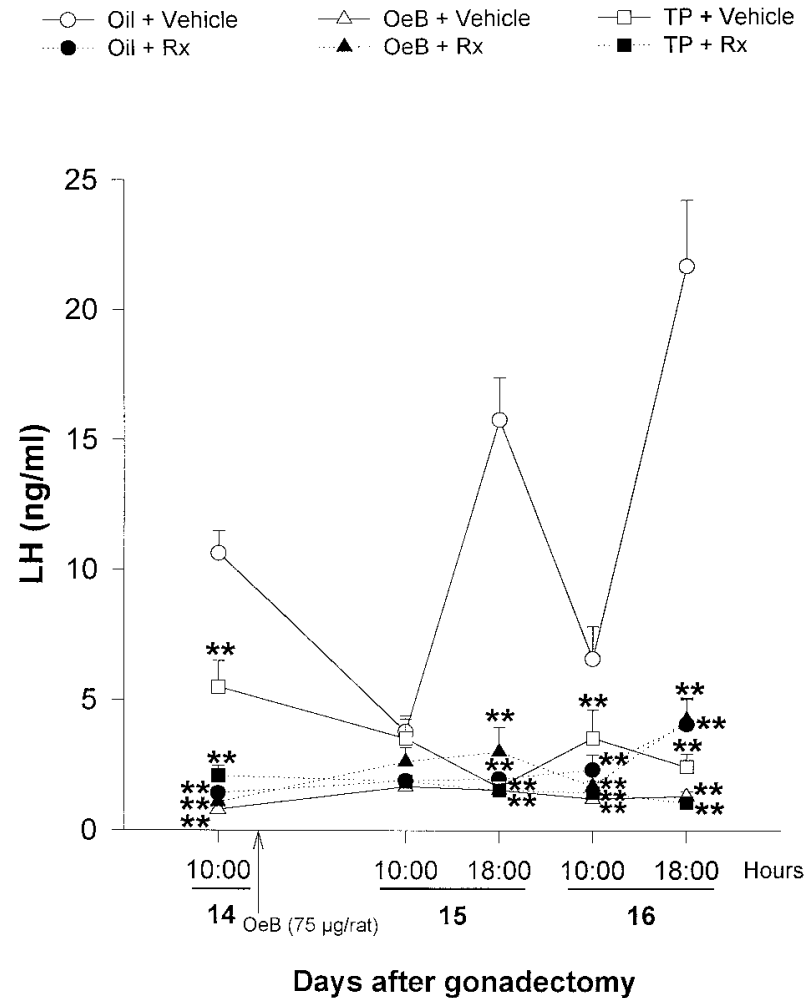

Figure 4 Serum LH concentrations in adult female rats treated with OeB $(100 \mu \mathrm{g} / \mathrm{rat})$, TP $(1 \cdot 25 \mathrm{mg} / \mathrm{rat})$ or oil on day 1 of age and with raloxifene $(500 \mu \mathrm{g} /$ rat per day) (solid symbols) or vehicle (open symbols) between days 1 and 5 of age. Blood samples were obtained on different days after ovariectomy (day 14 , $1000 \mathrm{~h}$; days 15 and 16, 1000 and $1800 \mathrm{~h}$ ). Animals were injected on day 14 post-ovariectomy with $75 \mu \mathrm{g} \mathrm{OeB} \mathrm{(1000} \mathrm{h).} \mathrm{Data} \mathrm{are}$ expressed as means \pm S.E.M. (9-11 animals per group). ${ }^{* *} P \leq 0 \cdot 01$ vs control group (ANOVA followed by Tukey's test).

Effects of neonatal raloxifene on adult $L H$ responses after administration of $75 \mu \mathrm{g} \mathrm{OeB}$ in female rats neonatally injected with $\mathrm{OeB}$ or TP (Fig. 4)

In control female rats, serum concentrations of $\mathrm{LH}$ were higher on day 14 post-ovariectomy than in rats neonatally injected with $\mathrm{TP}, \mathrm{OeB}$ or raloxifene. In oestrogenized and androgenized female rats, administration of $75 \mu \mathrm{g} \mathrm{OeB} \mathrm{did}$ not significantly change $\mathrm{LH}$ concentrations at any time studied. In these animals, raloxifene was ineffective in normalizing the LH response after administration of $75 \mu \mathrm{g}$ OeB.

\section{Discussion}

Neonatally administered steroids act at central as well as on peripheral targets. Of the peripheral actions, vaginal opening is one of the most commonly monitored markers. Vaginal opening is advanced after neonatal administration 
of oestradiol (Kincl et al. 1965, Feder 1967) and blocked after administration of testosterone (Tramezzani et al. 1963). The present results are merely confirmative of these well-known observations. In addition, as a first observation, the two SERMs used, tamoxifen and raloxifene, showed different activities, since advancement in vaginal opening was observed after raloxifene administration, but not after tamoxifen treatment (Table 1), which indicates an oestrogen-like action of raloxifene on vaginal development in agreement with previous data using 100, 250 and $500 \mu \mathrm{g} /$ day raloxifene (Pinilla et al. 2001a). This oestrogenic action of raloxifene is supported by the inhibition in LH secretion detected at vaginal opening.

The mechanism for sexual differentiation of the brain in rodents is still a matter of debate. The major question that remains to be solved is whether brain development in the female takes place in the complete absence of oestrogens (Gorski 1990). To clarify this, the administration of antioestrogens or ovariectomy during the critical differentiation period have been used as experimental approaches. In female rats treated with oestrogens or antioestrogens neonatally, sterility was observed in adulthood (Gorski 1963, Gorski \& Wagner 1965, Döhler \& Hancke 1978, Hancke \& Döhler 1980). In contrast, neonatal ovariectomy did not change the normal functional differentiation of the female brain (Gorski \& Wagner 1965). These apparently contradictory studies suggest either that a certain degree of exposure to oestrogens is necessary to normal differentiation or, alternatively, that the brain is inherently feminine or, if undifferentiated, it has the potential to become female in the absence of steroid signals (Gorski 1990). In the present study, we aimed to obtain additional information on the effects of neonatal administration of ICI 182,780 (a pure antioestrogen) (Wakeling et al. 1991, Wakeling \& Bowler 1992, Wade et al. 1993), tamoxifen and raloxifene (compounds with a mixed oestrogenic/ antioestrogenic activity) on the organization of reproductive function in female rats.

The present results confirm that administration of high doses of $\mathrm{OeB}$ or TP permanently alters the normal differentiation of the brain, and reinforce the hypothesis that oestrogens are needed for normal brain differentiation in female rats, since administration of a pure antioestrogen, such as ICI 182,780 , permanently altered the function of the reproductive axis. In addition, our current data show that the two SERMs used (raloxifene and tamoxifen) induced different peripheral actions (raloxifene advanced vaginal opening whereas tamoxifen did not), while their actions on brain differentiation appear to be similar. Finally, raloxifene was unable to block the effects of OeB and TP administered neonatally.

The alterations induced by neonatal administration of TP or OeB (acyclicity, anovulation, ovarian atrophy, and loss of negative and positive feedback between oestradiol and $\mathrm{LH}$ ) are in accordance with a number of previous studies (Barraclough 1961, Gorski 1963, Mennin \& Gorski 1975, Aguilar et al. 1983). In addition, postnatal treatment of female rats with antioestrogens, such as ICI-628 or MER-25, has been shown to interfere with hypothalamic feminization (McEwen et al. 1977, Döhler \& Hancke 1978) and to prevent hypothalamic masculinization by oestrogens or androgens (Döhler \& Hancke 1978). Our present results indicate that ICI 182,780 at the dose used did not affect either the age of vaginal opening or $\mathrm{LH}$ concentrations at that stage, thus suggesting a short-time action for this compound. In contrast, adult reproductive function was affected, since $46 \%$ of females neonatally treated with ICI 182,780 showed constant vaginal oestrus and changes in the control of $\mathrm{LH}$ secretion, as evidenced by the reduced response to ovariectomy (Fig. 1), and the absence of positive feedback between oestradiol and $\mathrm{LH}$ (Fig. 2). These results confirm that normal hypothalamic differentiation in females requires a certain degree of oestrogenic input.

Despite the fact that divergent peripheral effects were observed for the SERMs under analysis, both tamoxifen and raloxifene strongly interfered with hypothalamic differentiation as permanent alterations in vaginal smears and ovarian atrophy were detected in all treated animals (Table $2)$. These findings were accompanied by a significant reduction in the negative feedback between oestradiol and LH (Fig. 1), as well as in the absence of positive feedback (Fig. 2).

Notably, a number of differences can be pointed out between the effects of ICI 182,780 and the two SERMs: (1) the percentage of animals with permanent changes in vaginal smears was significantly greater after administration of SERMs than after administration of ICI 182,780, (2) the degree of ovarian atrophy was also higher in animals treated with SERMs than in those treated with ICI 182,780 and (3) the LH response to ovariectomy was more severely altered in animals treated with SERMs (Fig. 1).

Although theoretically these differences may be explained by considering the three drugs as antioestrogens with different potency, it is likely that the effects of tamoxifen and raloxifene (at the doses tested) were exerted through an oestrogenic activity of these compounds upon hypothalamic neurones when administered neonatally. In favour of this hypothesis are the following facts: (1) perinatal treatment of male rats with tamoxifen caused reproductive tract lesions and sexual behaviour deficits similar to those induced by diethylstilboestrol (DES; a synthetic compound with oestrogenic activity) (Vancutsem \& Roessler 1997), (2) tamoxifen administered neonatally showed uterotrophic action similar to DES (Poulet et al. 1997), (3) the effects of raloxifene and oestradiol given neonatally to female and male rats were similar (Pinilla et al. 2001a), (4) raloxifene showed, in adult ovariectomized females, an oestrogen-like action evidenced by the reduction in $\mathrm{LH}$ secretion and the 
increase of prolactin release (Pinilla et al. 2001b) and (5) raloxifene was unable to block the effects of oestradiol and testosterone given neonatally.

Since, in the CNS, testosterone is transformed into oestradiol (Reddy et al. 1974, Weisz \& Gibbs 1974), the effects of neonatally administered testosterone upon the organization of hypothalamic function seem to be dependent on its aromatization to oestradiol. Dihydrotestosterone (a non-aromatizable androgen) is unable to affect reproductive function (Whalen \& Luttge 1971, McDonald \& Doughty 1972, 1974), and the effects of testosterone were blocked by inhibitors of aromatization (Vreeburg et al. 1977) and antioestrogens (McDonald \& Doughty 1972). The analysis of the effects of combined administration of testosterone and oestradiol with raloxifene supports the oestrogenic activity of raloxifene. If raloxifene acts as an antioestrogen on the CNS a protective action against the effects of testosterone or oestradiol would be expected. On the contrary, some effects of testosterone (e.g. the degree of ovarian atrophy, the blunted LH response to ovariectomy) were reinforced by raloxifene. The effects of administration of oestradiol alone or oestradiol plus raloxifene were similar, which also indicate that, in the present experimental paradigm, raloxifene is devoid of an antioestrogenic action. The possibility that, with other treatment regimens, raloxifene may act as an antioestrogen requires further experimental work.

We have previously demonstrated that raloxifene administered to adult ovariectomized females decreases LH secretion and increases prolactin release, thus suggesting an oestrogenic action (Pinilla et al. 2001b). However, such an oestrogenic activity may become evident in the absence of endogenous oestrogens, since raloxifene implanted in the hypothalamus attenuates the positive feedback of oestradiol on LH secretion (Petersen \& Barraclough 1989, Petersen et al. 1989), blocks the pituitary effects of oestradiol on LH secretion (Simard \& Labrie 1985) and diminishes the effects of oestradiol on the expression of progesterone receptor mRNA in rat brain (Shughrue et al. 1997). Our present results demonstrated that, in the neonatal period, raloxifene was unable to counteract the effects of oestradiol and testosterone. It is possible that the effects of raloxifene during the neonatal period showed differences from those observed in adulthood. Since the mechanisms whereby SERMs carry out oestrogenic and antioestrogenic activities in different tissues or functions are not clearly understood at the present, it is possible that oestrogenic activity of raloxifene and tamoxifen may be fully expressed during the process leading to hypothalamic differentiation during the neonatal period. Further experimental work is necessary to clarify this point.

In conclusion, present experiments confirm that normal hypothalamic differentiation of the female rat requires a certain degree of oestrogenic input, and indicate that administration of tamoxifen and raloxifene at the present doses during the critical period of neonatal differentiation exert an oestrogenic action on the differentiation of the hypothalamic network responsible for the control of reproductive function in the female rat.

\section{Acknowledgements}

The authors are indebted to Rocío Campón for her excellent technical assistance. This work was supported by grants from DGESIC (Ministerio de Educación y Cultura, Spain) and project 1FD97-0696-02 (FEDER).

\section{References}

Aguilar E, Fernandez-Galaz C, Tejero A \& Vaticón MD 1979 PRL response to LHRH in neonatally estrogen treated female rats. Acta Endocrinologica (Suppl) 225197.

Aguilar E, Fernandez-Galaz C, Vaticón MD, Tejero A \& Aguilar E 1983 Oestrogen-bromocriptine interaction in the control of luteinizing hormone and prolactin secretion in the neonatally oestrogenized female rat. Journal of Endocrinology 97 319-325.

Barraclough CA 1961 Production of anovulatory sterile rats by single injection of testosterone propionate. Endocrinology 68 62-67.

Brown-Grant K 1974 Failure of ovulation after administration of steroid hormones and hormone antagonists to female rats during the neonatal period. Journal of Endocrinology 62 683-684.

Delmas PD, Barnason NH, Mitlak BH, Ravoux A, Shah AS, Huster WJ, Draper MW \& Christiansen C 1997 Effects of raloxifene on bone mineral density, serum cholesterol concentration and uterine endometrium in postmenopausal woman. New England Journal of Medicine 337 1641-1647.

Dhar JD \& Setty BS 1976 Epididymal response to exogenous testosterone in rats sterilized neonatally by estrogen. Endocrinologie 68 14-41.

Döhler KD \& Hancke JL 1978 Thoughts on the mechanism of sexual brain differentiation. In Hormones and Brain Development, pp 153-156. Eds G Dörner \& M Kawakami. Amsterdam: Elsevier.

Feder HH 1967 Specificity of testosterone and estradiol in the differentiating neonatal rat. Anatomical Record 157 79-86.

Glasebrook AL, Phillis DL \& Sluka JP 1993 Multiple binding sites for the anti-estrogen raloxifene (LY 56758). Journal of Bone and Mineral Research 8 (Suppl 1) A607.

Gorski RA 1963 Modification of ovulatory mechanism by postnatal administration of estrogen to the rat. American Journal of Physiology 205 842-844.

Gorski RA 1990 Sexual differentiation of the brain: comparative aspects. In Control of the Onset of Puberty, pp 231-250. Eds ML Grumbach, PC Sizoneko \& ML Aubert. Baltimore: Williams \& Wilkins.

Gorski RA \& Wagner JW 1965 Gonadal activity and sexual differentiation of the hypothalamus. Endocrinology 76 226-239.

Hancke JL \& Döhler KD 1980 Postnatal estradiol treatment prevents tamoxifen-induced defeminization of the female rat brain. Acta Endocrinologica 234 102-103.

Jordan VC 1993 A current view of tamoxifen for the treatment and prevention of breast cancer. British Journal of Pharmacology 110 507-517.

Jordan VC \& Murphy CS 1990 Endocrine pharmacology of antiestrogens as antitumor agents. Endocrine Reviews 11 578-610 .

Jordan VC \& Morrow M 1999 Tamoxifen, raloxifene and prevention of breast cancer. Endocrine Reviews 20 253-278. 
Kincl FA, Folch PA, Maqueo M, Herrera LL, Oriol A \& Dorfman RI 1965 Inhibition of sexual development in male and female rats treated with various steroids at the age of five days. Acta Endocrinologica 49 193-206.

McDonald PG \& Doughty C 1972 Comparison of the effect of neonatal administration of testosterone and dihydrotestosterone in the female rat. Journal of Reproduction and Fertility 30 55-62.

McDonald PG \& Doughty C 1974 Effect of neonatal administration of different androgens in the female rat: correlation between aromatization and the induction of sterilization. Journal of Endocrinology 61 95-103.

McEwen BS, Lieberburg I, Chaptal C \& Krey LC 1977 Aromatization: important for sexual differentiation of the neonatal rat brain. Hormones and Behaviour 9 249-263.

Mennin SP \& Gorski RA 1975 Effects of ovarian steroids on plasma LH in normal and persistent estrous adult female rats. Endocrinology 96 486-491.

Petersen SL \& Barraclough CA 1989 Suppression of spontaneous LH surges in estrogen treated ovariectomized rats by microimplants of antiestrogens into the preoptic brain. Brain Research 10 279-289.

Petersen SL, Cheuk C, Hartman R \& Barraclough CA 1989 Medial preoptic microimplants of the antiestrogen, keoxifene, affect luteinizing hormone-releasing hormone mRNA levels, median eminence luteinizing hormone-releasing hormone concentrations and luteinizing hormone release in ovariectomized, estrogen-treated rats. Journal of Neuroendocrinology 1 279-283.

Pinilla L, Trimiño E, Garnelo P, Bellido C, Aguilar R, Gaytan F \& Aguilar E 1993 Changes in pituitary secretion during the early postnatal period and anovulatory syndrome induced by neonatal oestrogen or androgen in rats. Journal of Reproduction and Fertility 97 $13-20$.

Pinilla L, Gonzalez LC, Gaytan F, Tena-Sempere M \& Aguilar E 2001a Oestrogenic effects of neonatal administration of raloxifene on hypothalamic-pituitary-gonadal axis in male and female rats. Reproduction 21 915-924.

Pinilla L, Gonzalez LC, Tena-Sempere M \& Aguilar E 2001 b Evidence for an estrogen-like action of raloxifene upon the hypothalamic-pituitary unit: raloxifene inhibits LH secretion and stimulated PRL secretion in ovariectomized female rats. Neuroscience Letters 311 347-358.

Poulet FM, Roessler ML \& Vancutsem PM 1997 Initial uterine alterations caused by developmental exposure to tamoxifen. Reproductive Toxicology 11 815-822.

Reddy VVR, Naftolin F \& Ryan KJ 1974 Conversion of androstendione to estrone by neural tissues from fetal and neonatal rats. Endocrinology 94 117-121.
Shughrue PJ, Lane MV \& Merchenthaler I 1997 Regulation of progesterone receptor messenger ribonucleic acid in the rat medial preoptic nucleus by estrogenic and antiestrogenic compounds: an in situ hybridization study. Endocrinology 138 5476-5484.

Simard J \& Labrie F 1985 Keoxifene shows pure antiestrogenic activity in pituitary gonadotrophs. Molecular and Cellular Endocrinology 39 141-144.

Tramezzani JH, Voloschin LM \& Nallar R 1963 Effect of a single dose of testosterone propionate on vaginal opening in the rat. Acta Anatomica 52 244-251.

Vancutsem PM \& Roessler ML 1997 Neonatal treatment with tamoxifen causes immediate alterations of the sexually dimorphic nucleus of the preoptic area and medial preoptic area in male rats. Teratology 56 220-228.

Vreeburg JTM, van der Vaart DM \& van der Schoot P 1977 Prevention of central defeminization but not masculinization in male rats by inhibition neonatally of oestrogen biosynthesis. Journal of Endocrinology 74 375-382.

Wade GN, Blaustein JD, Gra JM \& Meredith JM 1993 ICI 182,780: a pure antiestrogen that affects behaviours and energy balance in rats without acting in the brain. American Journal of Physiology 265 1392-1398.

Wakeling AE \& Bowler J 1992 ICI 182,780, a new antioestrogen with clinical potential. Journal of Steroid Biochemistry and Molecuar Biology 43 173-177.

Wakeling AE, Valcaccia B, Newboult E \& Green LR 1984 Non-steroidal antiestrogens - receptor binding and biological response in rat uterus, rat mammary carcinoma and human breast cancer cells. Journal of Steroid Biochemistry 20 111-120.

Wakeling AE, Dukes M \& Bowler J 1991 A potent specific pure antiestrogen with clinical potential. Cancer Research 51 3867-3873.

Weisz J \& Gibbs C 1974 Conversion of testosterone and androstanedione to estrogens in vitro by the brain of female rats. Endocrinology 94 616-620.

Whalen RE \& Luttge WG 1971 Perinatal administration of dihydrotestosterone to female rats in the development of reproductive function. Endocrinology 89 1320-1332.

Yang NN, Venugopalan M, Hardikar S \& Glasebrook A 1996 Identification of an estrogen response element activated by metabolites of $17 \beta$-estradiol and raloxifene. Science 273 1222-1225.

Received in final form 16 November 2001 Accepted 23 November 2001 